\title{
La bonification du crédit favorise-t-elle vraiment le financement des exploitations agricoles ? Cas de la wilaya de Laghouat en Algérie
}

\author{
Farid Oubraham ${ }^{1,2, *}$, Slimane Bédrani ${ }^{2}$ et Fathi Abdellatif Belhouadjeb ${ }^{3,4}$ \\ ${ }^{1}$ Université Ammar Télidji, Laghouat, Algérie \\ 2 École nationale supérieure agronomique (ENSA), Alger, Algérie \\ ${ }^{3}$ Institut National de la Recherche Agronomique (INRA), Djelfa, Algérie \\ ${ }^{4}$ Centre de Recherche en Agropastoralisme (CRAPAST), Djelfa, Algérie
}

\begin{abstract}
Résumé - Les exploitations agricoles algériennes sont confrontées à d'importantes difficultés de financement qui limitent considérablement leur capacité de production. Leur difficulté d'accès au crédit bancaire est due en grande partie à l'indisponibilité d'informations fiables sur leur situation financière et à leur faible taux de remboursement. Pour inverser la tendance et faciliter l'accès des exploitants agricoles au crédit bancaire, les pouvoirs publics ont revisité l'usage de la bonification des taux d'intérêts en mettant en place pour eux en 2011 un crédit d'investissement totalement bonifié appelé Ettahadi. Cependant, l'intervention publique dans le système de crédit, à travers la bonification, n'a eu qu'un très faible effet sur la demande de financement et un effet mitigé sur l'accès effectif au crédit. Les résultats d'une enquête menée auprès d'un échantillon représentatif d'agriculteurs de la wilaya de Laghouat ainsi que l'analyse des données de la Banque de l'agriculture et du développement rural montrent que la gestion du risque de crédit et les objectifs de rentabilité ont poussé cette banque publique à adopter une forme organisationnelle qui limite la distribution de crédit.
\end{abstract}

Mots clés : financement agricole / crédit / bonification / asymétrie d'information

Abstract - Does interest rate subsidy really promote the financing of farms? The case of the wilaya of Laghouat in Algeria. Algerian farms are faced with significant financing difficulties, which considerably limit their production capacity. Their difficulty in accessing bank credit is largely due to the unavailability of reliable information on their financial situation and their low repayment rate. In 2011, to reverse the trend and facilitate farmers' access to bank credit, the public authorities have revisited the use of the interest rate subsidy by setting up in favor of farmers a fully subsidized investment loan called Ettahadi. However, public intervention in the credit system through the subsidy has had very little effect on the demand for finance and a mixed effect on actual access to credit. The results of a survey conducted among a representative sample of farmers in the wilaya of Laghouat as well as the analysis of data from the Bank of Agriculture and Rural Development show that the management of credit risk and its profitability objectives have pushed this public bank to adopt an organizational form that limits the distribution of credit.

Keywords: agricultural financing / credit / subsidy / information asymmetry

\section{Introduction}

\subsection{Le financement agricole, entre intervention et désengagement de l'État}

La question du financement agricole, son origine comme ses canaux, constitue une question essentielle et la façon de

*Auteur de correspondance : faridoubraham@yahoo.fr l'aborder a considérablement évolué depuis les années 19601970 dans l'ensemble des pays du Sud (Doligez et al., 2008). D'un point de vue historique, en effet, la vision de la problématique du financement agricole montre deux grandes tendances (Doligez et Gentil, 2000) : celle de l'intervention et celle du désengagement de l'État des systèmes de crédit. Pour la tendance dite «développementaliste», qui considère que l'objectif est la distribution de crédit au plus grand nombre et à moindre coût, le taux de remboursement n'est pas un problème 
prioritaire ; pour la tendance bancaire, en revanche, le crédit est une opération financière sérieuse qui doit être remboursé de façon à garantir la pérennité du système bancaire de crédit.

Cette différence d'approche demeure en grande partie d'actualité même si, comme le souligne Lapenu (2008), les approches du «tout État» dans les années 1960-1970 et celles des «marchés financiers ruraux » à partir des années 1980 ont montré leurs limites face aux défaillances de l'État d'un côté et à celles du marché de l'autre. Alors que l'offre de crédit par les banques est reconnue depuis longtemps comme déterminante pour la croissance (Assidon, 1996), à l'échelle mondiale une majorité de petits paysans n'y a pas accès (Banque mondiale, 2008, p. 170). En Algérie, où l'implication des banques dans le financement des activités agricoles demeure marginale (Daoudi et Wampfler, 2010), la proportion d'exploitants ayant accès au crédit était de l'ordre de $10 \%$ dans les années 2000 (Abdelhakim et al., 2011) et a chuté à 2,2\% en 2015 (Daoudi, 2016).

Tributaires en effet de leurs spécificités propres qui rendent leur financement difficile, risqué et coûteux (Wampfler et Lapenu, 2002; Desjardins, 2010), les exploitations agricoles accèdent difficilement au crédit bancaire, particulièrement en Algérie où elles se distinguent par l'opacité de leur gestion.

\subsection{Relation banque-exploitations agricoles et asymétrie d'information}

Lorsqu'une entreprise sollicite un prêt, elle livre de l'information dont la qualité se révèle essentielle pour la relation banque-entreprise (Allegret et Baudry, 1996). Si l'information n'est pas fiable, n'est pas vérifiable ou est inexistante, la relation banque-entreprise sera caractérisée par une asymétrie d'informations et les intérêts des parties en présence ne convergeront pas (Rivaud-Danset, 1991; Dhieux et al., 2015). Dans un tel contexte, il existe de fortes probabilités qu'une relation de crédit entraîne des phénomènes de sélection adverse et d'aléa moral (Stiglitz et Weiss, 1981). C'est pourquoi dans une relation de crédit, la première étape de la relation de financement pour la banque est constituée par l'acquisition d'informations au sujet de l'entreprise. Il s'agit d'identifier le niveau de risque associé à l'activité de celle-ci car, pour chaque niveau de risque, la banque offrira des conditions de prêt différentes (Bédué et Lévy, 1997), voire refusera l'octroi de crédit si elle ne dispose pas d'informations pertinentes sur l'emprunteur, à même de lui permettre d'apprécier correctement le risque. Dit autrement, le risque de non-remboursement du prêt est alors très élevé, ce qui constitue pour Hodgman (1960) la principale cause qui pousserait une banque à adopter une forme procédurale lui permettant de limiter la distribution de crédit, non pas par l'augmentation des taux d'intérêts mais par la limitation de la quantité offerte.

Cela est particulièrement vrai dans le contexte algérien, où les exploitations agricoles se caractérisent par une gestion opaque et où l'acquisition d'informations par la banque à leur sujet repose presque exclusivement sur l'information acquise lors de l'établissement de la relation de crédit. Dans ce contexte algérien où le crédit est peu développé, afin de susciter une implication plus importante de la Banque de l'agriculture et du développement rural (BADR) et l'accroissement du nombre d'exploitants agricoles éligibles au crédit institutionnel, les pouvoirs publics ont revisité l'usage de la bonification des taux d'intérêts en mettant en place en 2011 un crédit d'investissement totalement bonifié (à intérêts nuls) appelé Ettahadi. L'Algérie étant un pays musulman, les pouvoirs publics sont partis de l'hypothèse selon laquelle l'intérêt constituerait un frein important à la demande de crédit, non pas parce qu'il augmente le coût du crédit mais plutôt parce qu'il est religieusement illicite. Cependant, malgré la mise en place de la bonification, la demande et l'accès effectif des agriculteurs au crédit demeurent faibles.

Le thème du financement de l'activité agricole en Algérie étant encore très peu étudié, l'intervention publique, à travers l'usage de la bonification, soulève plusieurs questions. La principale est de savoir si, à elle seule, la bonification peut améliorer l'accès au crédit des exploitants, sachant que les facteurs à l'origine de leur exclusion du financement demeurent inchangés?

Cette question nous semble d'autant plus pertinente si l'on considère le fait que la BADR, banque publique, se trouve face à un choix difficile : celui de répondre aux objectifs politiques de l'État en adhérant au dispositif, comme le stipule la convention la liant au ministère de l'Agriculture et du Développement rural (MADR), et financer le plus grand nombre d'exploitants agricoles, ce qui pourrait compromettre sa rentabilité ; ou celui de préserver ses objectifs de rentabilité en refusant ou en limitant, autant que faire se peut, l'accès des agriculteurs au crédit.

Partant de l'hypothèse que la BADR opterait pour le second choix, cette étude vise, dans un premier temps, à mettre en évidence l'existence d'asymétries d'informations dans la relation BADR-exploitants agricoles, dans un deuxième temps, à analyser la logique des acteurs en présence en termes de demande de financement et d'accès au crédit, dans un troisième temps, à déterminer la stratégie et les outils utilisés par la BADR pour limiter l'accès des exploitants au crédit, et enfin dans un quatrième temps, à vérifier empiriquement l'effet des outils utilisés par la BADR pour limiter l'accès au crédit sur l'échantillon étudié.

\section{Matériels et méthodes}

\subsection{Contexte de l'étude: politique de mise en valeur des terres et financement agricole}

Historiquement, la wilaya de Laghouat était une contrée dédiée à l'élevage ovin extensif, d'où son appellation de «pays du mouton ». Majoritairement issues de l'application de la loi sur l'Accession à la propriété foncière agricole (APFA) de 1983, les exploitations agricoles de la wilaya de Laghouat ont, depuis leur création, été confrontées à d'importantes difficultés de financement. Ce qui expliquerait que, depuis 1984 et sur les 50000 hectares de terres attribuées, on enregistre un taux de mise en valeur de seulement $18 \%$, un taux de mise en culture très bas de $6 \%$ (Direction des services agricoles, 2016), et un fort taux d'abandon.

\subsection{Méthodologie, collecte des données et échantillonnage}

Les données de ce travail sont issues d'une enquête de terrain menée dans la wilaya de Laghouat $(400 \mathrm{~km}$ au sud 


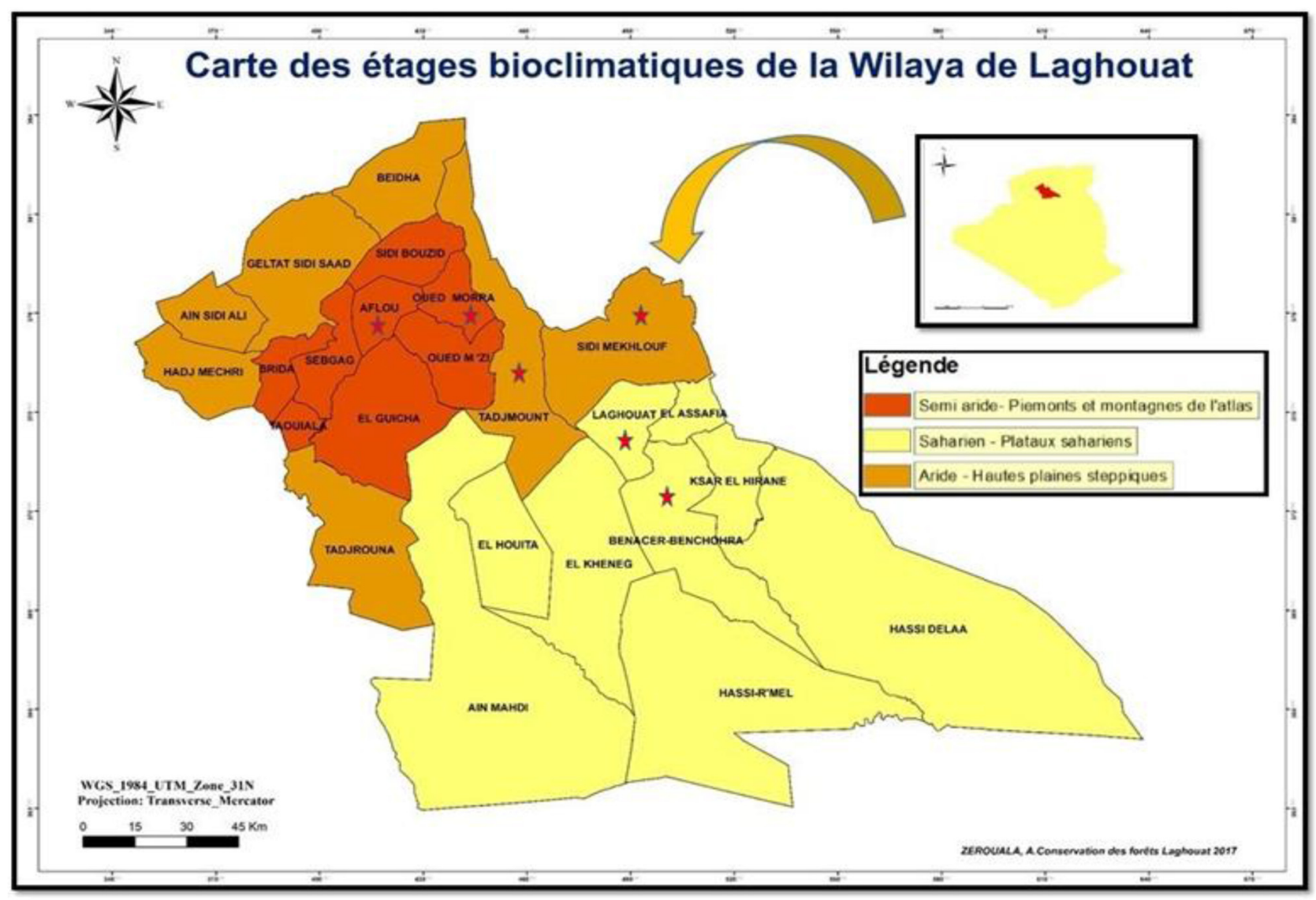

Source : conservation des forêts de la wilaya de Laghouat, modifiée (2020)

Fig. 1. Carte des étages bioclimatiques de la wilaya de Laghouat et position des 6 communes retenues ( $\star$ ). Source : conservation des forêts de la wilaya de Laghouat (2017), modifiée (2020).

Fig. 1. The bioclimatic levels map of the region of Laghouat.

d'Alger). Elle a été entamée en décembre 2017 et finalisée en décembre 2018. Quarante pour cent des demandeurs du crédit Ettahadi se concentrent dans 6 des 24 communes (marquées $\star$ sur la figure 1), mais ces communes sont équitablement réparties dans les étages bioclimatiques du territoire. Les $60 \%$ de demandeurs restant sont répartis à travers les 18 autres communes.

Nous avons donc eu recours à un choix d'échantillon raisonné à partir de la population mère des 6 communes (1210 exploitants) qui enregistrent le plus de demandes, en utilisant la formule de Bernoulli :

$$
n=\frac{N \cdot(t)^{2}}{(t)^{2}+\left[(l)^{2}(N-1)\right]},
$$

avec :

$-n=$ taille de l'échantillon à étudier;

$-N=$ taille de la population mère;

$-l=$ largeur de la fourchette égale à deux fois la marge d'erreur;

$-t=$ intervalle de confiance. Nous avons utilisé une probabilité de $99 \%$ et une marge d'erreur de $10 \%$.

Les calculs nous ont conduits à retenir un échantillon de 146 agriculteurs.
L'analyse des données a débuté par des statistiques descriptives. Puis les relations entre le non-accès au crédit et les variables explicatives ont été établies par régression logistique. Cette dernière vise à expliquer une variable d'intérêt binaire, c'est-à-dire de type " $1=$ accès au crédit» ou $« 0=$ non-accès au crédit». Elle permet de mesurer l'association entre la survenance d'un évènement (variable «expliquée» qualitative) et les facteurs susceptibles de l'influencer (variables «explicatives»).

L'une des principales limites de cette étude a été de se voir refuser par la BADR l'accès à beaucoup d'informations nécessaires à ce travail, comme la liste des demandeurs de crédits et leurs dossiers. Cela nous aurait permis de dégager quelques variables (le capital foncier dont dispose l'exploitant et la superficie cultivée, la dispersion géographique, l'âge de l'exploitant et de l'exploitation, la bancarisation, la relation de long terme de l'exploitant avec la banque, les motifs de rejet pour les agriculteurs non financés, les types d'élevages et de cultures pratiqués, le statut matrimonial, la pluriactivité, le nombre d'enfants à charge, etc.). Ce n'est qu'en septembre 2020 que les données issues de l'enquête ont été complétées par des données générales que la BADR a bien voulu nous céder lors des entretiens qu'elle nous a accordés. 
Tableau 1. Demandes satisfaites et non satisfaites en fonction des montants sollicités (en dinars).

Table 1. Satisfied and unsatisfied demands according to the requested amounts (in dinars) (Survey data).

\begin{tabular}{|c|c|c|c|c|c|c|c|c|}
\hline & \multicolumn{8}{|c|}{ Données de l'enquête pour la période 2011/2018 } \\
\hline & Effectif & $\%$ & Effectif & $\%$ & Effectif & $\%$ & Effectif & $\%$ \\
\hline Accès & 3 & 8,6 & 2 & 5,7 & 1 & 2,9 & 6 & 17,1 \\
\hline Non-accès & 6 & 17,1 & 18 & 51,4 & 5 & 14,3 & 29 & 82,8 \\
\hline Total & 9 & 25,7 & 20 & 57,1 & 6 & 17,2 & 35 & 100 \\
\hline
\end{tabular}

Source : établi à partir des données de l'enquête auprès des agriculteurs.

\section{Résultats}

\subsection{Asymétrie d'information banque-exploitants et bancarisation}

Si l'on considère la moyenne d'âge des exploitations agricoles étudiées qui est de 18 ans, celles-ci auraient donc pu accumuler sur cette période importante des informations sur leurs activités, comme l'évolution du chiffre d'affaires, de la production et de l'emploi (Adair et Adaskou, 2016). Toutefois, cela ne serait utile que si ces informations étaient à la fois disponibles, accessibles et vérifiables. Ce qui n'est pas le cas des exploitations étudiées. En effet, les données de l'enquête montrent qu'en raison de leur évolution dans un environnement quasi informel, les exploitations agricoles de la wilaya de Laghouat disposent de peu d'informations enregistrées. Aucune des exploitations ne dispose de documents comptables, certifiés ou non, ou de documents relatifs à la protection sociale des ouvriers agricoles permanents et/ou saisonniers, car ceux-ci ne sont pas déclarés. De plus, la rémunération des ouvriers agricoles se fait exclusivement en espèces et en nature.

Quant à la bancarisation des exploitants interrogés, c'est-àdire la proportion de la population titulaire d'un compte bancaire, elle est de l'ordre de $14 \%$, ce qui est relativement faible. Si de plus on considère l'utilisation effective du compte et des services financiers afférents, la bancarisation n'est qu'apparente puisque ce compte n'est jamais utilisé. En effet, toutes les transactions des exploitants se règlent exclusivement en espèces. Ils expliquent cette situation par le fait que l'utilisation du chèque comme instrument de paiement n'est pas une pratique courante et qu'il n'est pas accepté par les fournisseurs; ils précisent enfin que l'utilisation des espèces est plus pratique, plus simple et beaucoup plus efficace.

Ces résultats montrent que l'écrasante majorité des exploitants interrogés n'entretient pas de relations suivies avec la BADR.

\subsection{Bonification et logique des acteurs en présence}

\subsubsection{Adhésion des agriculteurs: demande de} financement

Pour la période 2011-2018, les données de l'enquête montrent que la proportion d'exploitants ayant sollicité un crédit d'investissement n'est que de $24 \%$. Du côté de la BADR, le nombre de demandes de financement enregistrées pour la même période n'est que de 92 (131 demandes au total en septembre 2020). Ramené aux 5950 exploitants recensés comme actifs dans la wilaya de Laghouat (Direction des services agricoles, 2019), ce taux n'est que de $2,2 \%$, ce qui est extrêmement faible.

Les exploitants n'ayant pas sollicité le crédit d'investissement, soit $76 \%$ de l'échantillon, expliquent ce comportement pour $31 \%$ par les difficultés pour obtenir le crédit (lourdeurs bureaucratiques et pots de vins, ce qui engendre d'importants coûts de transactions), pour $24 \%$ par leur non-connaissance de l'existence du crédit, et enfin pour $28 \%$ par des motifs religieux.

\subsubsection{Réponse de la BADR aux demandes de crédits}

\subsubsection{Accès au crédit en fonction des montants sollicités}

Les données de l'enquête (Tab. 1) montrent que les montants des crédits sollicités se situent à $26 \%$ entre 1 et 3 millions de dinars algériens ( $1 \mathrm{DA}=0,006 €$ en avril 2021), à $57 \%$ entre 4 et 10 millions de dinars et à $17 \%$ entre 12 et 75 millions de dinars. Elles montrent également que le taux d'accès au crédit n'est que de $17 \%$ et que le taux de satisfaction des demandes de crédit est plus élevé pour les exploitants ayant sollicité les crédits les plus faibles. En effet, $50 \%$ des exploitants ayant eu accès au crédit ont sollicité des crédits allant de 1 à 3 millions de dinars.

Les données de la BADR (Tab. 2) dessinent la même tendance et mettent en évidence un taux global d'accès au crédit de $33 \%$ et un taux de $79 \%$ pour les exploitants ayant sollicité des crédits allant de 1 à 3 millions de dinars.

L'analyse des données met en évidence un accès au crédit différencié selon les montants sollicités. Celles de la BADR, en particulier, montrent bien que le taux d'accès au crédit est négativement corrélé à l'importance du montant du crédit. À mesure que celui-ci augmente, le taux d'accès diminue. Autrement dit, la BADR semble privilégier l'accès au crédit des agriculteurs sollicitant les montants les plus faibles.

Enfin, l'examen de la situation du crédit de campagne appelé $R$ 'fig et également bonifié, confirme cette tendance et montre que le taux d'accès au crédit de campagne est plus important que celui de l'accès au crédit d'investissement. Il est en effet de $94,1 \%$ et de $67 \%$ respectivement selon les données de l'enquête et de celles de la BADR ( $c f$. Fig. 2).

Ces données montrent que la BDAR semble privilégier l'accès au crédit de court terme au détriment des crédits de moyen et long termes. Cela s'expliquerait par le fait que pour le 
Tableau 2. Demandes satisfaites et non satisfaites en fonction des montants sollicités (en dinars).

Table 2. Satisfied and unsatisfied demands according to the requested amounts (in dinars) (BADR data).

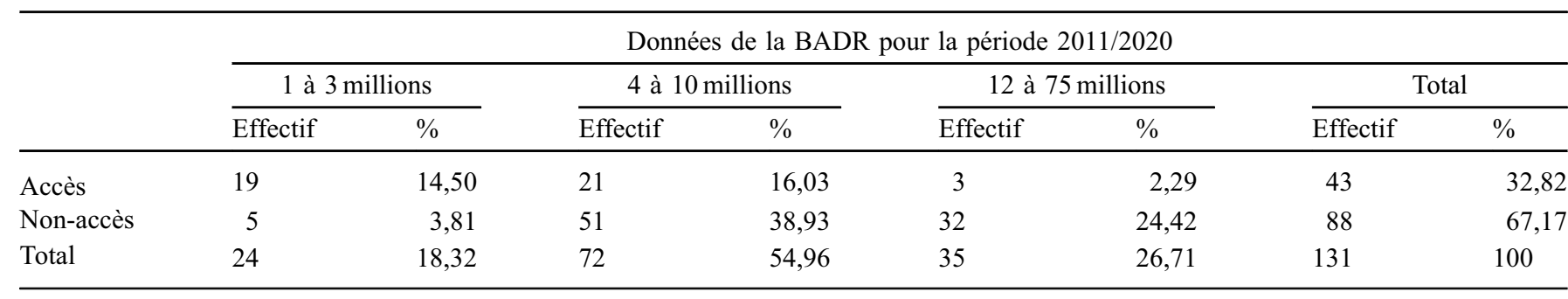

Source : établi à partir des données de la BADR.

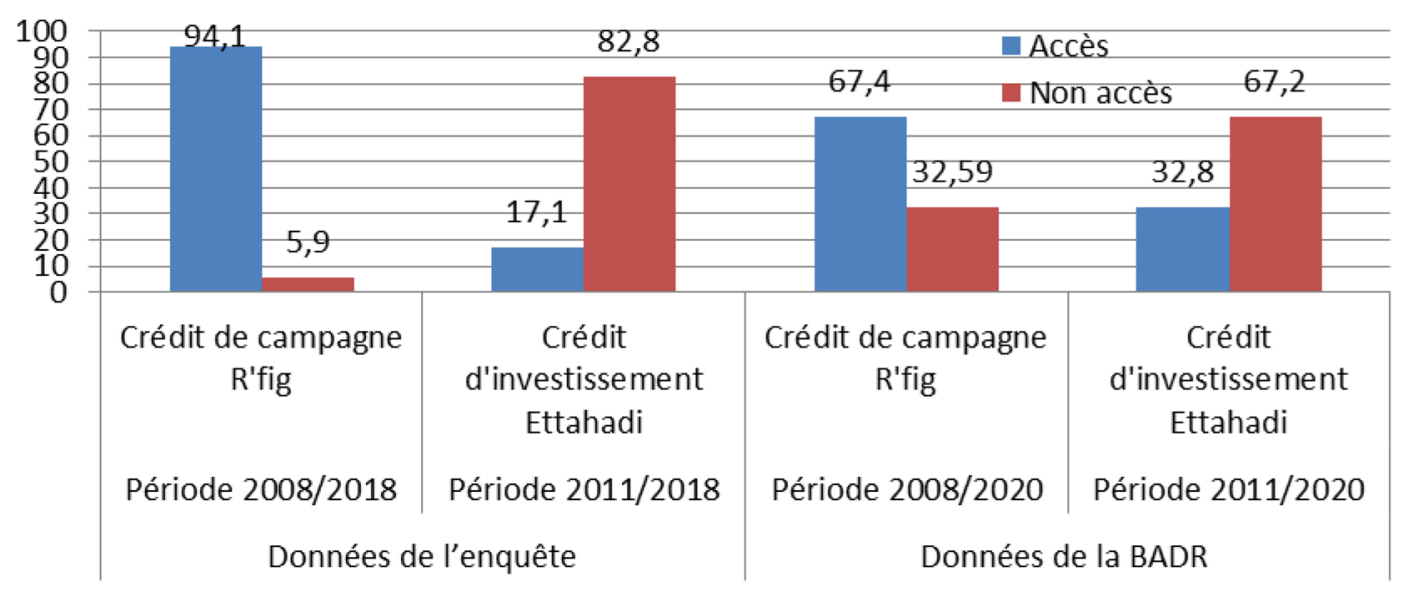

Source : établi à partir des données de l'enquête auprès des agriculteurs et de celles de la BADR Laghouat, 2020

Fig. 2. Taux des demandes satisfaites et non satisfaites en fonction du type de crédit.

Fig. 2. Rate of satisfied and unsatisfied demands depending on the type of credit.

crédit de campagne $R$ 'fig, la banque n'engage que de faibles capitaux (risques de pertes réduits) et pour des durées de crédit plus courtes (risques d'immobilisation plus faibles).

\subsubsection{Accès au crédit en fonction des délais de notification}

L'enquête auprès des agriculteurs montre des délais de notification de la BADR qui vont de 7 à 48 mois avec une majorité qui dépassent 18 mois. Cela expliquerait pourquoi plus de $95 \%$ des demandeurs du crédit d'investissement jugent la procédure compliquée et les délais trop longs, alors qu'à l'inverse, les demandeurs de crédit de campagne les jugent plutôt acceptables car la majorité ont accédé au crédit au plus tard au bout de 2 mois maximum, d'une part, et que ces délais n'ont aucune incidence sur le calendrier cultural, d'autre part, puisque les demandes de crédit sont formulées et satisfaites avant le début de la campagne agricole. Ce qui n'est pas le cas du crédit d'investissement pour lequel les délais longs se traduisent, selon les déclarations des agriculteurs concernés, par des surcoûts suite à la réévaluation de l'offre financière du fournisseur et des manques à gagner liés à une mise en place tardive des investissements. La question des délais ayant été soumise à la BADR, celle-ci nous a déclaré que, jusqu'en 2019, les dossiers de crédits déposés par les agriculteurs n'étaient pas reçus contre des accusés de réception. Dans pareille situation, non seulement l'exploitant se trouve dans l'incapacité de faire valoir ses droits, notamment en termes de délais réglementaires, mais de plus la voie est ouverte à toutes les dérives.

\subsubsection{Stratégie adoptée par la BADR pour limiter la distribution de crédit}

Pour la BADR, comme pour toute banque, accorder un crédit est une décision qui est à la fois irréversible et risquée, particulièrement dans un contexte où elle ne dispose pas d'informations sur la capacité et surtout sur la volonté de remboursement des exploitants. En effet, l'État ne prenant en charge, durant les trois premières années, que les intérêts des exploitants qui honorent les échéances de paiement, la BADR peut considérer que la bonification ne lui apporte aucune solution en cas de non-remboursement, car pour elle le problème des impayés éventuels demeure entier. De plus, habitués à voir leurs dettes épongées de façon récurrente par les pouvoirs publics, les agriculteurs ne sont pas particulièrement 
Tableau 3. Outils utilisés par la BADR pour limiter la distribution de crédit.

Table 3. Tools used by the BADR bank to limit the distribution of credit.

\begin{tabular}{|c|c|}
\hline Apport personnel & $\begin{array}{l}\text { Même si le règlement qui régit les opérations de crédit situe l'apport personnel entre } 10 \text { et } 15 \% \text { du } \\
\text { montant de l'investissement, dans le cas du crédit d'investissement Ettahadi la BADR Laghouat exige un } \\
\text { apport personnel qui se situe entre } 40 \text { et } 50 \% \text {. Dans la mesure où rares sont les exploitants qui disposent } \\
\text { d'un tel apport personnel, cette mesure, qui vise le partage du risque, a aussi pour effet d'évincer du crédit } \\
\text { un grand nombre d'exploitants }\end{array}$ \\
\hline Caution solidaire & $\begin{array}{l}\text { Pour réduire les risques, la BADR exige une caution solidaire. Pour la BADR, cela représente une garantie } \\
\text { d'exécution du contrat de crédit par une tierce personne en cas de défaillance de l'emprunteur, avec deux } \\
\text { avantages. Cela lui permet à la fois de recouvrer ses créances sans passer par une procédure judiciaire et de } \\
\text { limiter l'accès au crédit des exploitants, car dans la mesure où le cautionnaire court un risque en cas de } \\
\text { non-remboursement du crédit par le contractant, peu de personnes sont prêtes à prendre cet engagement }\end{array}$ \\
\hline $\begin{array}{l}\text { Types de cultures et } \\
\text { d'élevages projetés }\end{array}$ & $\begin{array}{l}\text { Sur } 95 \% \text { des crédits accordés, } 52 \% \text { portent sur l'acquisition de cheptel ovin, } 17 \% \text { sur l'acquisition de } \\
\text { cheptel bovin laitier, } 3 \% \text { sur la transformation de lait cru de vache et } 22 \% \text { sur la culture de céréales (blé } \\
\text { tendre et blé dur). Les différés de paiement sont presque exclusivement accordés aux exploitants dont les } \\
\text { investissements portent sur ces élevages et cultures. Cela pourrait s'expliquer par le fait que: } \\
\text { - l'élevage ovin, principale activité pratiquée dans la région, est à forte valeur ajoutée; de plus, la } \\
\text { demande en viande rouge ovine est très forte et stable, en raison notamment de sa consommation tout au } \\
\text { long de l'année (fêtes, funérailles, etc.); } \\
\text { - pour ce qui est de l'élevage bovin, les éleveurs, les collecteurs et les transformateurs de lait cru de vache } \\
\text { bénéficient de subventions publiques : } 12 \mathrm{DA} / \text { litre de lait produit, } 4 \mathrm{DA} / \text { litre de lait collecté et } 6 \mathrm{DA} / \text { litre de } \\
\text { lait transformé } \\
\text { - s'agissant des céréales, les producteurs bénéficient des prix garantis à la production : } 4500 \mathrm{DA} / \text { quintal de } \\
\text { blé dur et } 3500 \mathrm{DA} / \text { quintal de blé tendre } \\
\text { Les subventions constituent ainsi des revenus supplémentaires et stables améliorant de manière significative } \\
\text { la capacité de remboursement des exploitants agricoles }\end{array}$ \\
\hline Délais de notification & $\begin{array}{l}\text { Les demandes de crédit n'étant pas enregistrées contre accusés de réception, la BADR a toute latitude pour } \\
\text { allonger inconsidérément les délais, ce qui conduit à dissuader le plus grand nombre }\end{array}$ \\
\hline
\end{tabular}

Source : établi à partir de la synthèse des entretiens réalisés avec la banque BADR de Laghouat, 2020.

encouragés à rembourser leurs crédits. Les données de la BADR montrent en effet que le taux d'impayés était de $71 \%$ en 2014.

La synthèse des divers entretiens réalisés avec la BADR nous a permis d'identifier les principaux leviers utilisés par celle-ci pour limiter ses risques, et donc les crédits accordés; la conjugaison de leurs effets expliquerait le faible accès au crédit (Tab. 3 et 4 ).

Le tableau 4 ci-dessous reprend les variables ayant permis à la BADR de limiter l'accès au crédit.

$\mathrm{Au}$ final, la probabilité qu'un exploitant agricole puisse satisfaire simultanément toutes les conditions mises en place par la BADR est très faible, car chaque mesure prise par cette dernière exclut une catégorie particulière d'exploitants. L'effet conjugué de toutes les mesures conduit à l'éviction de plus de $67 \%$ des demandeurs de crédit.

\subsection{Déterminants de l'accès des exploitations agricoles au crédit}

Nous avons tenté de vérifier empiriquement l'effet des variables utilisées par la BADR sur l'accès au crédit des agriculteurs interrogés; nous leur avons ajouté d'autres variables citées régulièrement par la littérature comme ayant un effet sur l'accès au crédit. Pour ce faire, nous avons eu recours à la régression logistique avec le modèle Logit (Tab. 5).
Le $\mathrm{Khi}^{2}$ du modèle est de 17,251 avec une signification statistique de 0,069 , ce qui est inférieur à 0,1 (au seuil de significativité de $90 \%$ ). Cela indique que les 10 variables retenues sont très significatives. Le test du rapport de vraisemblance (-2log-vraisemblance) est égal à 14,819, le $\mathrm{R}^{2}$ de Cox et Snell est de 0,389 et celui de Nagelkerke est de 0,649 . Cela signifie que le poids conjugué des 10 variables explique bien le faible taux d'accès au crédit. Ainsi, la variabilité de l'exclusion du financement bancaire des exploitations est expliquée à $65 \%$. Le degré de concordance entre les valeurs prédites et les valeurs observées est de 91,4\% dans le modèle global.

Les rapports des cotes $\operatorname{Exp}(\mathrm{B})$ correspondent au nombre de fois d'appartenance à un groupe lorsque la valeur du prédicteur augmente de 1 . Plus précisément, un rapport des cotes plus grand que 1 indique une augmentation des chances de faire partie du groupe d'exploitants accédant au crédit, tandis qu'un rapport des cotes de moins de 1 diminue les probabilités d'appartenance à ce groupe.

Au seuil de significativité de $90 \%$, l'analyse des coefficients individuels des variables confirme l'effet de la stratégie adoptée par la BADR et montre qu'un agriculteur possédant un terrain en propriété, disposant d'au moins une source supplémentaire de revenu, ayant une expérience dans l'activité agricole, disposant d'une grande superficie et bien formé, a plus de chances d'accéder au crédit d'investissement. Le modèle montre aussi que la variable «âge» est inférieure à 
Tableau 4. Variables utilisées par la BADR pour limiter l'accès au crédit.

Table 4. Variables used by the BADR bank to limit the access to credit.

Les garanties

Âge de l'exploitant

Superficie

Accès au crédit de campagne $R^{\prime} f i g$

Antécédents de non-remboursement

Autre source de revenu

Nombre d'années d'expérience
L'État exige de la banque le financement des exploitations agricoles en propriété et en concession. Or, les concessions, qui demeurent propriété de l'État, sont inaliénables et, de ce fait, ne peuvent constituer une garantie réelle pour la BADR. 20\% des demandeurs de crédit ont des actes de concessions

Les règlements de la banque stipulent que l'âge limite pour la demande d'un crédit s'établit à $70 \mathrm{ans}$; mais dans la pratique, cette limite a été ramenée à 60 ans. Les données de la BADR montrent que $78 \%$ de l'accès au crédit concerne les 39-59 ans, dont $75 \%$ concerne les $40-59$ ans

La BADR exige une superficie moyenne de 10 hectares. Pour la BADR, une superficie relativement importante générerait plus de profit, d'une part, et augmente la valeur de la garantie, d'autre part. Or $34 \%$ des demandeurs de crédit disposent d'une superficie comprise entre 2 et 5 hectares

Les exploitants agricoles ayant sollicité le crédit de campagne bonifié $R$ 'fig ont été favorisés. Cela pourrait s'expliquer par le fait que l'asymétrie d'informations entre la BADR et ces exploitants est faible car la BADR dispose d'informations à leur sujet Les exploitants agricoles n'ayant pas remboursé leurs crédits antérieurs sont systématiquement exclus de tout nouveau crédit

Les exploitants disposant de revenus supplémentaires extra-agricoles sont favorisés pour l'accès au crédit

Les exploitants ayant plusieurs années d'expérience dans l'activité agricole sont favorisés pour l'accès au crédit

Source : établi à partir de la synthèse des entretiens réalisés avec la banque BADR de Laghouat, 2020.

Tableau 5. Résultats du modèle logit d'analyse des déterminants de l'accès au crédit.

Table 5. Results of the Logit model for analyzing the determinants of access to credit.

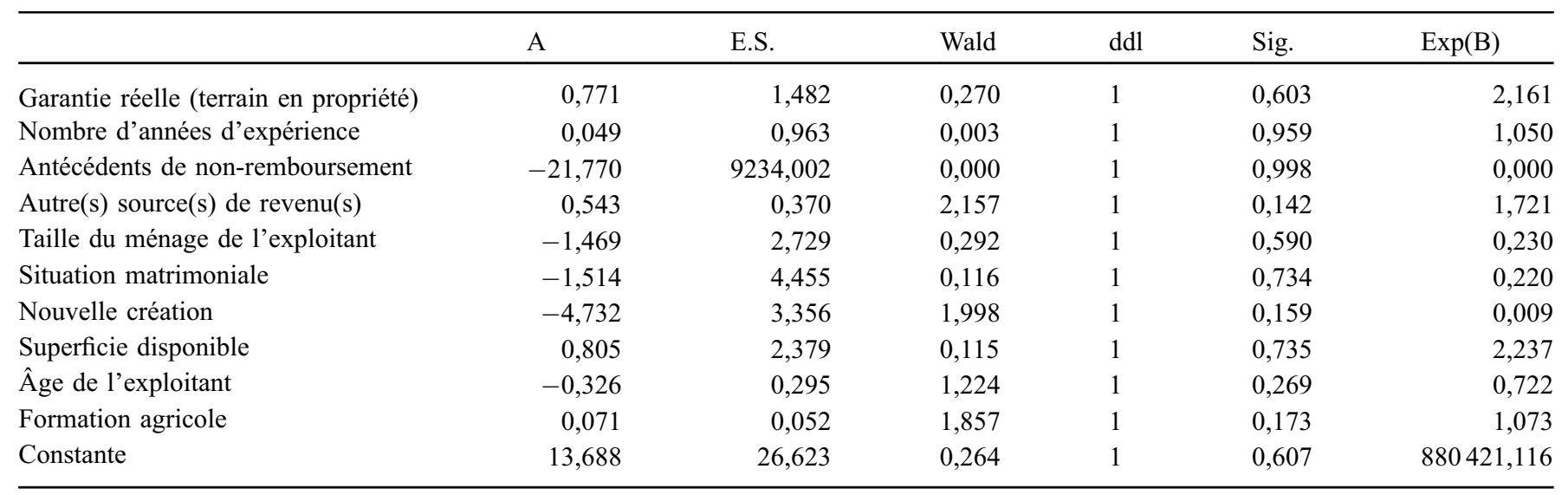

A : coefficient du modèle; E.S. : estimation; Wald : test de Wald; ddl : degré de liberté ; Sig : significativité ; Exp(B) : rapports des cotes. Source : auteurs, 2020.

1, ce qui veut dire qu'il y a un âge optimal auquel la probabilité d'obtenir du crédit est la plus élevée; le modèle le situe entre 40 et 50 ans. Ainsi, sachant que la moyenne d'âge de la population étudiée est de 59 ans et que près de $71 \%$ des exploitants ont un âge compris entre 51 et 76 ans, une bonne partie des agriculteurs ont donc de fortes chances de se voir exclus du financement.

Le modèle montre qu'un agriculteur débiteur vis-à-vis de la banque, ou ayant pour but la création d'une exploitation agricole, se verrait exclu de l'accès au crédit. Le fait d'être célibataire ou marié avec une taille de ménage élevée semble ne pas avoir d'effet sur l'accès au crédit.

\section{Conclusion}

L'ambition de cette étude était de vérifier l'hypothèse selon laquelle la bonification des taux d'intérêts n'aurait pas d'effet significatif sur une implication plus importante de la BADR dans le financement des exploitations agricoles de la wilaya de Laghouat et donc sur leur accès au crédit. Se trouvant face à l'opacité des exploitations agricoles (peu de données disponibles) et à l'obligation de préserver ses objectifs de rentabilité, la BADR a adopté une forme procédurale qui lui permet de limiter l'accès des agriculteurs au crédit. 
Sans mesures complémentaires de nature, notamment, à réduire les asymétries d'informations caractérisant la relation banque-exploitant, la politique de bonification des taux d'intérêts est manifestement loin de constituer un outil efficace d'incitation à l'utilisation du crédit bancaire et à l'amélioration du taux d'accès des agriculteurs à celui-ci. Si la bonification a pour effet de réduire le coût du crédit, elle ne peut cependant pas favoriser l'accès des exploitations agricoles au crédit bancaire si les facteurs à l'origine de leur exclusion du financement demeurent inchangés d'une part et si le système de financement n'est pas adapté aux activités agricoles d'autre part.

Ces résultats soulèvent ainsi des questions relatives à l'intervention publique et plus précisément aux processus de leur élaboration, à leur pertinence et à leur efficacité/efficience, car malgré les nombreuses interventions de l'État algérien dans l'agriculture, la question de la sécurité alimentaire constitue encore aujourd'hui, et probablement pour les décennies à venir, un véritable défi pour le pays et son agriculture. Relever ce défi suppose une réelle volonté politique qui doit déboucher sur la mise en place d'un système de crédit à la fois concerté et adapté aux spécificités des activités agricoles, aux attentes des producteurs et aux caractéristiques de leurs exploitations (petite taille, faiblesse du capital propre).

La problématique de financement d'un secteur aussi stratégique que l'agriculture pour un pays comme l'Algérie demeure donc entièrement posée, et dépasse largement la seule question de la bonification des taux d'intérêt.

\section{Références}

Abdelhakim T, Bessaoud O, Dolle V. 2011. Repenser le financement agricole en Afrique du Nord au service de tous les agriculteurs. In: Financement du développement agricole et rural en Méditerranée, lettre de veille $\mathrm{n}^{\mathrm{o}} 17$, juin 2011. Montpellier: CIHEAM-IAM.

Adair P, Adaskou M. 2016. Les déterminants du rationnement du crédit des PME en France : un modèle de déséquilibre sur un panel cylindré (2002-2010). In: $2^{\mathrm{e}}$ Colloque international sur la défaillance d'entreprises (CIDE), octobre 2016, Nanterre, France, 16 p.

Allegret JP, Baudry B. 1996. La relation banque-entreprise : structures de gouvernement et formes de coordination. Revue française d'économie 11(4): 3-36. https://doi.org/10.3406/rfeco.1996.1007.

Assidon E. 1996. L'approfondissement financier : épargne et crédit bancaire. Revue Tiers Monde 37(145): 153-171. https://doi.org/ 10.3406/tiers.1996.5036.

Banque mondiale. 2008. L'agriculture au service du développement. Rapport sur le développement dans le monde. Banque mondiale, 394 p. https://doi.org/10.3917/dbu.banqu.2008.01.
Bédué A, Lévy N. 1997. Relation banque-entreprise et coût du crédit. Revue d'économie financière 39: 179-200. https://doi.org/10.3406/ ecofi.1997.2297.

Daoudi A, Wampfler B. 2010. Le financement informel dans l'agriculture algérienne: les principales pratiques et leurs déterminants. Cahiers Agricultures 19(4): 243-248. https://doi. org/10.1684/agr.2010.0414.

Daoudi A. 2016. Les leçons du fonctionnement du financement informel en Algérie. Grain de sel 72: 20-21. https://www.interreseaux.org/wp-content/uploads/gds72_financement_informel. pdf.

Desjardins, Développement international. 2010. Financement agricole : un puissant outil de contribution à la sécurité alimentaire des populations. Québec, Canada, 24 p. https://www.findevgateway. org/sites/default/files/publications/files/mfg-fr-publications-diver ses-financement-agricole-et-securite-alimentaire-des-populations2010.pdf.

Direction des services agricoles. 2016. Situation du secteur agricole de la wilaya de Laghouat. Algérie: Direction des services agricoles, $18 \mathrm{p}$.

Direction des services agricoles. 2019. Situation du secteur agricole de la wilaya de Laghouat. Algérie: Direction des services agricoles, $33 \mathrm{p}$.

Dhieux A, Severin E, Vigneron L. 2015. Qualité de l'information comptable et recours au crédit fournisseur. GERME - Groupe d'études et de recherches en management des entreprises EA $\mathrm{n}^{\circ} 4112$, 30 p. https://hal.inria.fr/hal-01188869/.

Doligez F, Gentil D. 2000. Le financement de l'agriculture. Partie 332 du Memento de l'agronome, document de travail, version 2. $22 \mathrm{p}$. https://www.iram-fr.org/ouverturepdf.php?file= 228.pdf.

Doligez F, Lemelle JP, Lapenu C, Wampfler B. 2008. Financer les transitions agricoles et rurales. Défis agricoles africains. Paris (France): Éditions Karthala, 146 p.

Hodgman DR. 1960. Credit risk and credit rationing. The Quarterly Journal of Economics 74(2): 258-278. https://doi.org/10.2307/ 1884253.

Lapenu C. 2008. Évolutions récentes dans l'offre et les stratégies de financement du secteur rural : échanges d'expériences et synthèse bibliographique. Rapport du Réseau français de la microfinance, sous-commission $\mathrm{n}^{\mathrm{o}} 3,147 \mathrm{p}$.

Rivaud-Danset D. 1991. La relation banque-entreprise : une approche comparée. Revue d'économie financière 16: 105-118. https://doi. org/10.3406/ecofi.1991.2411.

Stiglitz JE, Weiss A. 1981. Creditrationing in markets with imperfect information. The American Economic Review 71(3): 393-410.

Wampfler B, Lapenu C. 2002. Le financement de l'agriculture familiale dans le contexte de libéralisation: quelle contribution de la microfinance. Résumé exécutif. pp. 21-24. https://agritrop.cirad. $\mathrm{fr} / 511653 /$.

Citation de l'article : Oubraham F, Bédrani S, Belhouadjeb FA. 2021. La bonification du crédit favorise-t-elle vraiment le financement des exploitations agricoles? Cas de la wilaya de Laghouat en Algérie. Cah. Agric. 30: 23. 